\title{
INVARIANT MANIFOLDS
}

BY M. W. HIRSCH, C. C. PUGH AND M. SHUB

Communicated by Stephen Smale, April 29, 1970

0 . Introduction. Let $M$ be a finite dimensional Riemann manifold without boundary. Kupka [5], Sacker [9], and others have studied perturbations of a flow or diffeomorphism of $M$ leaving invariant a compact submanifold. Anosov [2] considers perturbations of a nonsingular flow, which of course leaves invariant each leaf of the foliation by trajectories. In both problems there is an assumption of hyperbolicity in planes normal to the submanifold, or trajectories.

We present a more general theory of diffeomorphisms hyperbolic to a compact laminated subset $\Lambda \subset M$. (This includes flows, by considering the time one map.) We suppose $\Lambda$ is the disjoint union of injectively immersed submanifolds, called leaves, whose tangent planes vary continuously on $\Lambda$. The diffeomorphism is assumed to permute the leaves, and its differential is more hyperbolic normal to the leaves than tangent to them. The main theorems assert that this situation persists under small perturbations of the diffeomorphisms. By means of the technical device of unwinding the leaves of $\Lambda$, the proofs are reduced to the case of a single invariant, closed submanifold.

Applications are made to stability of group actions and $\Omega$-stability. See also references [4] through [8].

1. Definition of $r$-hyperbolicity. Let $E \rightarrow B$ be a vector bundle with a Finsler structure, and $T: E \rightarrow E$ a linear bundle map covering $\phi: B \rightarrow B$. Define

$$
\rho(T)=\limsup _{n \rightarrow \infty} \sup _{x \in B}\left\|\left(T^{n}\right)_{x}\right\|^{1 / n}
$$

Let $L \subset E$ be a vector subbundle invariant under $T$. Call $T r$ normally hyperbolic (or simply $r$-hyperbolic) to $L, r \in \boldsymbol{Z}_{+}$, if $T$ is a homeomorphism, and there is a splitting $E=N_{+} \oplus L \oplus N_{-}$invariant under $T$, such that

$$
\rho\left(T \mid N_{-}\right)<\min \left\{1, \rho(T \mid L)^{r}\right\}
$$

and

AMS 1969 subject classifications. Primary 3465, 2240, 3451, 3453, 5736, 5482.

Key words and phrases. Invariant manifolds, foliation, perturbation of flow, perturbation of diffeomorphism, structural stability, topological transitivity. 


$$
\rho\left(T^{-1} \mid N_{+}\right)<\min \left\{1, \rho\left(T^{-1} \mid L\right)^{r}\right\} .
$$

This means that under iteration $T$ contracts every vector of $N_{-}$more than $T^{i}$ contracts any vector of $L$, while $T$ expands every vector of $N_{+}$more than $T^{i}$ expands any vector of $L_{+}$, for $i=0,1, \cdots, r$.

Let $V \subset M$ be a submanifold. A diffeomorphism $f:(M, V) \rightarrow(M, V)$ is $r$-hyperbolic to $V$ if the bundle map $T f: T_{V} M \rightarrow T_{V} M$ is $r$-hyperbolic to $T V$.

2. Invariant submanifolds. Let $f: M \rightarrow M$ be a diffeomorphism. For $A \subset M$ define a $A$-stable and $A$-unstable manifolds of $f$ to be the respective sets:

$$
W_{A}^{s}(f)=\bigcap_{n \geqq 0} f^{-n}(A),
$$

and

$$
W_{A}^{u}(f)=\bigcap_{n \geqq 0} f^{n}(A)=W_{A}^{8}\left(f^{-1}\right) .
$$

2.1. THEOREM. Let $V \subset M$ be a compact $C^{1}$ submanifold and $f:(M, V) \rightarrow(M, V)$ a diffeomorphism which is $r$-normally hyperbolic to $V$. Then $V$ is $C^{r}$ and there exists a neighborhood $A \subset M$ of $V$ such that $W_{A}^{u}(f)$ and $W_{A}^{s}(f)$ are $C^{r}$ submanifolds of $M$ intersecting in $V$, tangent along $V$ to $N_{+} \oplus T V$ and $N_{-} \oplus T V$ respectively. If $x \in W_{A}^{s}(f)$, there exists $y \in V$ such that $\lim _{n \rightarrow \infty} d\left(f^{n} x, f^{n} y\right)=0$. If $x \in W_{A}^{n}(f)$, there exists $z \in V$ such that $\lim _{n \rightarrow \infty} d\left(f^{-n} x, f^{-n_{z}}\right)=0$.

The proof is by successive approximations. Let $X \subset M$ be a trial submanifold through $V$, tangent along $V$ to $N_{+} \oplus T V$. The sequence of submanifolds $\left\{f^{n}(X) \cap A\right\}_{n \in Z_{+}}$converges to a topological submanifold, which is $W_{A}^{u}(f)$; the argument is similar to the case $V=a$ point in $[3, \S 2]$. The smoothness of $W_{A}^{u}(f)$ is proved by a simplification of 2.3 of [3].

The same construction also works for perturbation of $f$, proving the following:

2.2. Theorem. Suppose $r \in \boldsymbol{Z}_{+}$. The neighborhood $A$ in 2.1 can be chosen so that there exists a $C^{1}$ neighborhood $\Re \subset \operatorname{Diffr}^{2}(M)$ of $f$, and a continuous map $h: \mathfrak{N} \rightarrow C^{r}(V, M)$, such that if $f^{\prime} \in \mathfrak{N}$, then

(a) $W_{A}^{s}\left(f^{\prime}\right)$ and $W_{A}^{u}\left(f^{\prime}\right)$ are $C^{r}$ submanifolds of $M$ intersecting transversely in a $C^{r}$ submanifold $V^{\prime}$,

(b) $f^{\prime}\left(V^{\prime}\right)=V^{\prime}$,

(c) $h\left(f^{\prime}\right)$ maps $V$ diffeomorphically onto $V^{\prime}$, and $h(f)=1_{V}$. 
3. Laminated subsets. A $C^{r}$-lamination of a subset $\Lambda \subset M$ is a function $\mathscr{L}$ assigning to each $x \in \Lambda$ a subset $\mathfrak{L}_{x} \subset \Lambda$, called the leaf through $x$, satisfying for all $x, y \in \Lambda$ :

(1) $x \in \mathfrak{L}_{x}$.

(2) There exists a connected $k$-manifold $V_{x}$ and an injective $C^{r}$ immersion $i_{x}: V_{x} \rightarrow M$ with $i_{x}\left(V_{x}\right)=\mathfrak{L}_{x}$.

(3) Either $\mathfrak{L}_{x}=\mathfrak{L}_{y}$ or $\mathfrak{L}_{x} \cap \mathfrak{L}_{y}=\varnothing$.

(4) There exists a neighborhood $U \subset \Lambda$ of $x$ and a continuous map $\phi: U \rightarrow C^{r}\left(D^{k}, M\right)$ such that $\phi_{x}:\left(D^{k}, 0\right) \rightarrow\left(\mathscr{L}_{x}, x\right)$, where $D^{k} \subset R^{k}$ is the closed unit ball, and $\phi x$ is a smooth embedding. We call $\phi$ a local chart for $\mathcal{L}$.

Let $P_{k}(M)$ be the bundle of $k$-planes tangent to $M$. The map $T \mathfrak{L}: \Lambda \rightarrow P_{k}(M)$, assigning to $x$ the $k$-plane $T \mathfrak{L}_{x}$ tangent to $\mathfrak{L}_{x}$ at $x$, is well defined and continuous. The union of these planes is a subbundle to $T_{V} M$, also denoted by $T \&$.

If $\Lambda=M$ then $\mathcal{L}$ is a foliation in the sense of Anosov [2].

A diffeomorphism $f$ of $M$ is an automorphism of $\mathscr{L}$ provided $f(\Lambda)=\Lambda$ and $f \circ \mathfrak{L}=\mathfrak{L} \circ f$, i.e., $f$ takes leaves on to leaves. If in addition $T f$ is $r$-hyperbolic to $T \mathscr{L}$, we say $f$ is $r$-hyperbolic to $\mathscr{L}$.

We write $h:\left(\Lambda_{1}, \mathscr{L}^{1}\right) \approx\left(\Lambda_{2}, \mathscr{L}^{2}\right)$ if $\mathscr{L}^{i}$ is a lamination of $\Lambda_{i}$ and and $h: \Lambda_{1} \rightarrow \Lambda_{2}$ is a homeomorphism such that $\mathscr{L}^{2} \circ h=h \circ \mathfrak{L}^{1}$.

Let $\Lambda / \mathcal{L}$ denote the space of leaves, with the usual quotient topology.

3.1. Theorem. Let $\&$ be a $C^{r}$-lamination of a compact set $\Lambda \subset M$. Assume either (1) the map $T \mathscr{L}$ is Lipschitz, or (2) $\Lambda / \mathfrak{L}$ is Hausdorff. Let $f: M \rightarrow M$ be a $C^{r}$ diffeomorphism which is $r$-hyperbolic to L. Given $\epsilon>0$ there is a $C^{1}$ neighborhood $\Re \subset \operatorname{Diff}^{r}(M)$, and a continuous map $h: \Re \rightarrow C^{0}(\Lambda, M)$, with the following properties. For each $f^{\prime} \in \mathfrak{N}$ there exists a $C^{r} k$-lamination $\mathcal{L}^{\prime}$ of a compact set $\Lambda^{\prime} \subset M$ such that

(a) $f^{\prime}$ is $r$-hyperbolic to $\mathcal{L}^{\prime}$;

(b) $h\left(f^{\prime}\right):(\Lambda, \mathfrak{L}) \approx\left(\Lambda^{\prime}, \mathcal{L}^{\prime}\right)$;

(c) $h(f)=1_{\mathscr{L}}$;

(d) $\lim _{f^{\prime} \rightarrow f} T \mathscr{L}^{\prime} \circ h\left(f^{\prime}\right)=T \mathscr{L}$ in $C^{0}\left(\Lambda, P_{k}(M)\right)$.

Theorem 3.1, applied to the case of a fibration, is used to prove the following result; see also [1]. Let $T^{4}$ be the 4-torus.

3.2. Theorem. $\operatorname{Diff}^{1}\left(T^{4}\right)$ contains a nonempty open set of diffeomorphisms which are topologically transitive but not structurally stable.

The idea of the proof of 3.1 is to construct a manifold $Q$ of the same dimension as $M$, a closed submanifold $V$ and a $C^{r}$ immersion $i: Q \rightarrow M$ mapping $V$ injectively onto $\Lambda$. There is a neighborhood $Q_{0} \subset Q$ of $V$ 
and a $C^{r}$ embedding $F: Q_{0} \rightarrow Q$ with $F(V)=V$, such that $i \circ F=f \circ i$. By abuse of language, $F$ is $r$-hyperbolic to $V$. Although $V$ might have uncountably many noncompact components, the compactness of $\Lambda$ enables us to prove analogues of 2.1 and 2.2 for $F$. Every sufficiently $C^{1}$ small perturbation $f^{\prime}$ of $f$ gives rise to a perturbation $F^{\prime}$ of $F$ such that $i \circ F^{\prime}=f^{\prime} \circ i$. We obtain an $F^{\prime}$-invariant submanifold $V^{\prime} \subset Q_{0}$ and a homeomorphism $H: V \rightarrow V^{\prime}$ which is uniformly $C^{r}$ close to $1_{V}$. We define $\Lambda^{\prime}=i\left(V^{\prime}\right)$ and $h=i \circ H \circ i^{-1} \mid \Lambda: \Lambda \rightarrow \Lambda^{\prime}$. The hyperbolicity of $f$ and assumption about $\mathscr{L}$ are used to prove $h$ injective. Stable and unstable manifolds of leaves of $\mathcal{L}$ are also obtained via $i$; they form $C^{r}$ laminations.

Although $h$ will not generally be smooth, it will be $C^{r}$ on each leaf, with continuous derivatives on $\Lambda$. More precisely

3.3. Theorem. Let $\phi: U \rightarrow C^{r}\left(D^{k}, M\right)$ be a local chart for $\mathfrak{L}$ with $U$ compact. Then each map $\psi\left(f^{\prime}, x\right)=h\left(f^{\prime}\right) \circ \phi_{x}: D^{k} \rightarrow M$ is $C^{r}$, the map $\psi: \Re \times U \rightarrow C^{r}\left(D^{k}, M\right)$ is continuous, and $\psi(f, x)=\phi_{x}$ for all $x \in U$.

If $\Lambda=M, 3.1$ has another addendum:

3.4. Theorem. Suppose $\Lambda=M$ in 3.1. There exists $\epsilon>0$ such that if $f^{\prime} \in \mathfrak{N}$, then $\mathscr{L}^{\prime}$ is the unique $C^{\mathbf{1}} k$-lamination of $M$ which is invariant under $f^{\prime}$, and which satisfies $d\left(T \mathfrak{L}, T \mathfrak{L}^{\prime}\right)<\epsilon$ in $C^{0}\left(M, P_{k}(M)\right)$.

4. Anosov actions. Let $G$ be a $k$-dimensional Lie group with a locally free $C^{r}$ action $\alpha$ on $M, r \geqq 2$. The components of orbits form a $C^{r} k$-lamination $\mathcal{L}^{\alpha}$ of $M$ such that $T \mathcal{L}^{\alpha}$ is $C^{1}$. We call $\alpha$ structurally stable if $T \mathscr{L}^{\alpha}$ has a neighborhood $\mathcal{U} \subset C^{1}\left(M, P_{k}(M)\right)$ such that for any locally free $C^{2}$ action $\beta$ with $T \mathscr{L}^{\beta} \in \mathcal{U}$, there is a homeomorphism

$$
h:\left(M, \mathcal{L}^{\alpha}\right) \approx\left(M, \mathfrak{L}^{\beta}\right) .
$$

The action $\alpha$ is called Anosov, or hyperbolic, if there exists $g \in G$, called an Anosov element, such that $g^{\alpha}$ is 1 -hyperbolic to $\mathfrak{L}^{\alpha}$.

4.1. Theorem. Anosov actions on compact manifolds are structurally stable.

ProOF. If $T \mathscr{L}^{\beta}$ is sufficiently $C^{1}$ close to $T \mathscr{L}^{\alpha}, 3.1$ applies with $f=g^{\alpha}, f^{\prime}=g^{\beta}$. By 3.4, the lamination $\mathscr{L}^{\prime}$ obtained from 3.1 must equal $\mathscr{L}^{\beta}$ if $T \mathscr{L}^{\beta}$ is sufficiently $C^{0}$ close to $T \mathscr{L}^{\alpha}$. Thus the homeomorphism $h\left(f^{\prime}\right)$ of 3.1 satisfies $(1)$.

\section{REFERENCES}

1. R. Abraham and S. Smale, Nongenericity of $\Omega$-stability (to appear).

2. D. V. Anosov, Geodesic flows on closed Riemannian manifolds of negative curva- 
ture, Trudy Mat. Inst. Steklov $90(1967)=$ Proc. Steklov Inst. Math. 90 (1967). MR 36 \#7157.

3. M. Hirsch and C. Pugh, Stable manifolds and hyperbolic sets, Proc. Sympos. Pure Math., vol. 14, Amer. Math. Soc., Providence, R. I., 1970.

4. M. Hirsch, Foliations and noncompact transformation groups, Bull. Amer. Math. Soc. 76 (1970), 1020-1023.

5. I. Kupka, Stabilité des variêtés invariantes d'un champ de vecteurs pour les petites perturbations, C. R. Acad. Sci. Paris 258 (1964), 4197-4200. MR 28 \#5238.

6. C. Pugh and M. Shub, Some more smooth ergodic actions (in preparation).

7. - , , - -stability for flows (in preparation).

8. —_ Linearizing normally hyperbolic diffeomorphisms and flows (in preparation).

9. R. Sacker, $A$ perturbation theorem for invariant Riemannian manifolds, Proc. Internat. Sympos. Differential Equations and Dynamical Systems (Mayaguez, P. R., 1965) Academic Press, New York, 1967, pp. 43-54. MR 36 \#1784.

University of California, Berkeley, California 94720 